\title{
COVID-19 impact on colleagues in the powder diffraction community
}

The COVID-19 virus pandemic has and will continue to have major impacts to all. We all hope the medical, scientific, and governmental leadership communities find ways to reduce the impacts and in time to find cures and vaccines that will greatly reduce the impact to individuals, the economy, and the advancement of all the sciences around the world. It is heartening to read about the rapid determination of the structure and the base sequences of the proteins in the COVID-19 virus. Based on the knowledge of the structure and the base sequences investigators have used supercomputers to computationally test millions of molecules for their potential to bind at key locations within the virus. Hopefully, several molecules suggested by the computational screenings will be found to effectively block the virus from attaching to human cells. The very quick sequencing of the proteins of the virus has also led to multiple companies around the world developing possible vaccines and launching testing programs. Clearly, the structural sciences, that many of Powder Diffraction's readers contribute to, have been exceptionally active and hopefully will soon lead to the development of means to reduce the health impacts of those infected and ideally develop vaccines for widespread immunization.

The impact of the virus on the Powder Diffraction community is also noted in terms of changes in plans for various conferences and short courses worldwide. Do make use of the two Calendar of Events contributions to PDJ to keep abreast with the status of planned conferences, schools, and workshops. In many cases, a change to virtual meeting format will require us to become comfortable with new methods to present and to learn about advances in our field. While I will greatly miss the face-to-face gatherings, I will be registering for several "virtual" conferences and aim to gain as much as I can from the "new" format of our long-standing technical conferences.
From my role as the Editor-in-Chief for Powder Diffraction I observed that in March and April there was a surge in papers being submitted to the Journal followed by a worrisome drop in submitted papers in May and June. I am very concerned that the virus' impact will take many months to several years for recovery of the researchers that are doing fundamental research in methods, developing computational tools, improving reference patterns and calibration standards, and studying materials ranging from pharmaceuticals to new battery cathodes and anodes, to metal-organic frameworks and all other types materials the PDJ community studies.

This issue of Powder Diffraction (PDJ) has a broad range of quality Technical Articles ranging from the certification of the next Si Standard Reference Material, to testing a flow-through capillary system for the study of re-solvation processes in pharmaceutical compounds, to extension of the deconvolution method for improving the data collected on Bragg-Brentano diffractometers, and to demonstration of an alternative neutron diffractometer arrangement for strain measurements. There are also several papers related to the crystal structure determination and correlation with spectra and properties. This issue of PDJ also contains three New Diffraction Data and Data Report Articles that provide high-quality powder diffraction reference data of important pharmaceutical compounds. The issue closes with an International Report on the TMS 2020 Annual Meeting \& Exhibition and perhaps very timely the Calendars of Forthcoming Meetings and of upcoming Short Courses and Workshops. 